\title{
Metrological Applications of X-ray Waveguide Thin Film Structures in X-ray Reflectometry and Diffraction
}

\author{
J.B. PeŁKa ${ }^{a}$ AND S. Lagomarsino ${ }^{b}$ \\ ${ }^{a}$ Institute of Physics, Polish Academy of Sciences \\ al. Lotników 32/46, 02-668 Warsaw, Poland \\ ${ }^{b}$ IESS CNR, V. Cineto Romano 42, 00156 Roma, Italy
}

\begin{abstract}
The effect of resonance, observed in X-ray waveguide layered structures in a characteristic way influences the scattering properties of the films. It is well known that in the resonant region the reflectivity shows a series of minima, usually very deep and extremely narrow. The positions and depths of the minima depend only on X-ray waveguide structural properties, on the $\mathrm{X}$-ray wavelength and on the incident beam divergence. In the present work we propose and discuss the application of the $\mathrm{X}$-ray waveguide and quasi $\mathrm{X}$-ray waveguide film structures as tools to experimental evaluation of some quantities related to X-ray reflectometric or diffractometric measurements, like the beam divergence, wavelength, or angular distance. Examples of application of the X-ray waveguide as an excellent tool to estimate the effective beam divergence are shown. Properties of the $\mathrm{X}$-ray waveguide elements as a handy wavelength or angular calibration standard are also mentioned.
\end{abstract}

PACS numbers: 68.65.-k, 61.10.Kw, 06.30.Bp

\section{Introduction}

X-ray waveguide (XWG) layered structures are extensively studied for a few recent years, mainly due to their ability of compressing the primary monochromatic synchrotron beam to nanometric sizes [1-3]. Their properties result from the resonance-enhanced propagation of the X-rays in thin films deposited on an $\mathrm{X}$-ray reflecting mirror, which was anticipated theoretically a decade ago $[4,5]$. The effect of resonance occurs whenever a particular combination of layer thickness, its electron density (with respect to deeper neighboring layer) and wavelength of the excited X-ray beam is obeyed. The resonant region occurs within the angles 
of incidence between $\theta_{c 1}$ and $\theta_{c 2}$, characterizing the critical angles of low density upper film and heavy X-ray mirror beneath, respectively.

The effect strongly influences the reflectivity of XWG samples, which shows in the resonant region a series of minima, first 2-3 being usually very deep and narrow. To obtain this type of reflectivity it is not necessary, as it is in the case of a true XWG structure that the low density film was covered with an ultra-thin layer of higher electron density. The reflectivity of quasi-XWG structures without this top coverage is characterized by similar properties. The positions and depths of the minima depend only on the specified above XWG structure and X-ray wavelength. An additional factor influencing the shapes (depths) of the minima is the effective instrumental broadening (c.f. Figs. 1 and 2).

Although in principle the theoretical divergence of an X-ray device can be calculated from geometrical dependences and properties of optical elements, like mirrors, monochromators, slits, etc., our knowledge of status of a particular system is often insufficient to do this correctly. Therefore, there is a strong need to find experimentally the value of the effective beam divergence, necessary for interpretation of recorded results. The existence of mentioned above strongly pronounced and very sharp structures in reflectivity of the XWG can be used not only in estimation of the divergence, but also can be helpful in accomplishing the wavelength or angular calibration in commonly used X-ray devices.

\section{Properties of the $X W G$ reflectivity}

If we write the index of refraction of the film as $n_{\text {ref }}=1-\delta+\mathrm{i} k$, the internal angle of incidence, $\theta_{\text {int }}$, can be expressed as

$$
\theta_{\text {int }}=\sqrt{\theta_{\mathrm{ext}}^{2}-2 \delta}
$$

Here $\theta_{\text {ext }}$ is the external angle of incidence.

Assuming the simplest geometrical condition for the angular position of the $n$-th resonance (reflectivity minimum) excited by monochromatic X-ray beam of wavelength $\lambda$ in a film of thickness $d_{0}$ to be $n \lambda=2 d_{0} \sin \theta_{n}$ [3], we get the following formula for the approximate positions of the resonant minima in the reflectivity of XWG:

$$
\left[\sin ^{-1}\left(\frac{n \lambda}{2 d_{0}}\right)\right]^{2}+2 \delta=\theta_{n}^{2} .
$$

The $\theta_{n}$ positions obtained using the above approximation differ from the exact values, which can be derived using the formula given by De Boer [5], who obtained for the positions of interference minima the following dependence:

$$
n \lambda=2 d_{0} N_{j z}^{\prime} \approx 2 d_{0} \sin \theta_{n}\left(1-\delta_{j} / \sin ^{2} \theta_{n}\right), \quad \text { if } \quad \theta_{n} \gg \theta_{c} .
$$

Here $N_{j z}^{\prime}$ is the $z$-th component real part of the complex vector of refraction, $\boldsymbol{N}_{j}$, defined as $\boldsymbol{N}_{j}=\boldsymbol{N}_{j}^{\prime}-\mathrm{i} \boldsymbol{N}_{j}^{\prime \prime}=\lambda \boldsymbol{k}_{j} / 2 \pi$, where $\boldsymbol{k}_{j}$ is the wave vector. 


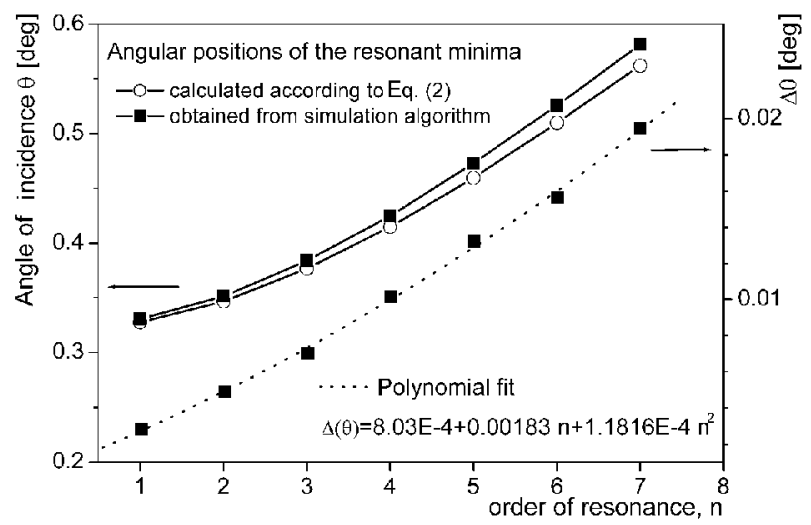

Fig. 1. Angular positions of reflectivity minima of the structure composed of $95 \mathrm{~nm}$ graphite deposited on nickel. Open circles - calculated according to Eq. (2). Full squares - obtained from the simulation algorithm. The differences in the exact and the approximate angular positions can be accurately fitted using a binomial (dotted line).

The calculation of the $\mathrm{X}$-ray reflectivity for the arbitrary multilayer film system can be precisely performed using the recursive algorithm introduced by Parratt [6], and based on Fresnel formula. Comparison of the angular positions of reflectivity minima of simple quasi-XWG structure calculated according to Eq. (2) and simulated using Parratt algorithm is shown in Fig. 1. The difference in the angular positions of the minima is not large, and can be accurately fitted to the positions obtained from the Parratt algorithm using a binomial.

\section{1. $X W G$ as a beam divergence probe}

Figure 2 shows an experimental reflectivity spectrum for the true waveguide structure (of composition Cr $5.5 \mathrm{~nm} / \mathrm{C} 74.3 \mathrm{~nm} / \mathrm{Au} 30 \mathrm{~nm} / \mathrm{Cr} 20 \mathrm{~nm} /$ Si substrate). It was recorded with monochromatic synchrotron beam of energy $13 \mathrm{keV}$ and divergence of $5 \mu \mathrm{rad}$ (about $3 \times 10^{-40}$ ). Thin solid line shows calculated reflectivity for the structure assuming zero roughness and a parallel beam. The theoretical depth in the first resonance reaches a value under $5 \%$ of incident beam intensity. This comparison shows that information about the beam divergence can be easily extracted fitting the experimental reflectivity with theoretical calculation assuming the divergence as a parameter.

Also the quasi-XWG structures, simplified to a thin film (usually made of graphite) of appropriately chosen thickness deposited on heavier substrate can be used for this purpose. In Fig. 3 the experimental reflectivity and the theoretical simulation for the structure composed of $98.5 \mathrm{~nm}$ graphite film (with an ultra-thin Ti layer in its center) deposited on Ni mirror are compared. The measurements were performed with conventional Cr X-ray tube. The positions of all structures observed in experimental reflectivity are in excellent agreement with theoretical 


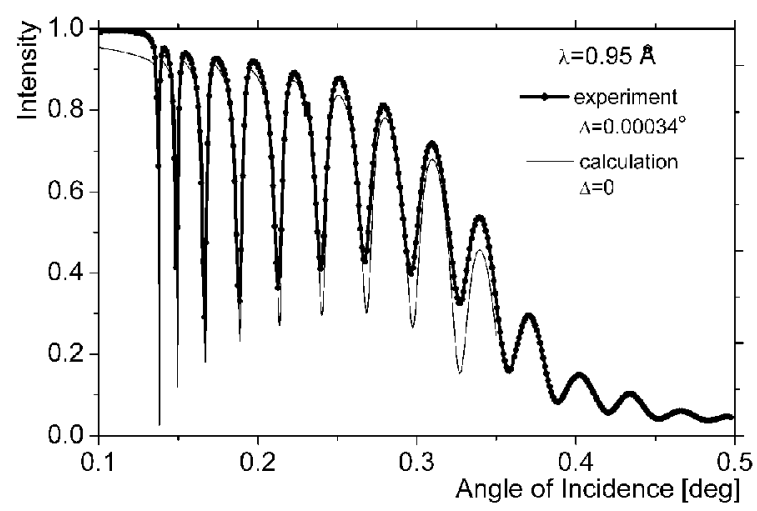

Fig. 2. Reflectivity of an X-ray waveguide structure of composition $5.5 \mathrm{~nm} \mathrm{Cr} /$ $74.3 \mathrm{~nm} \mathrm{C} / 30 \mathrm{~nm} \mathrm{Au} / 20 \mathrm{~nm} \mathrm{Cr} / \mathrm{Si}$ substrate. Points - experiment with monochromatic synchrotron beam of energy $13 \mathrm{keV}(\lambda=0.95 \AA)$, size of $3 \mu \mathrm{m}$, and divergence of $5 \mu \mathrm{rad}$ (about $0.0003^{\circ}$ ). Thin solid line - calculated reflectivity for the structure, assuming zero roughness and parallel beam. FWHM of the first experimental minimum was found to be $0.0012^{\circ}$.

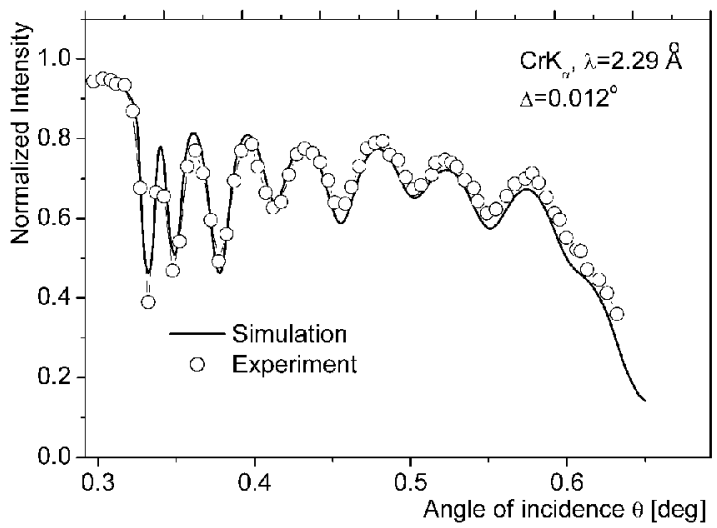

Fig. 3. Reflectivity of quasi-X-ray waveguide structure composed of a $98.5 \mathrm{~nm}$ thick graphite film with an ultra-thin Ti layer $0.5 \mathrm{~nm}$ thick in its center, deposited on $82 \mathrm{~nm}$ $\mathrm{Ni}$ mirror. Calculations were performed assuming $0.7 \mathrm{~nm}$ roughnesses on all interfaces and for the $5414 \mathrm{eV}$ photon energy (Cr $K_{\alpha}$ radiation).

predictions. It is evident that the magnitude of suppression of experimental depth in the resonant minima also in this case can be used to extract the information about the effective divergence of the primary beam. This suppression decreases with the order of minimum, mainly due to the fact that the natural width of the minimum increases with the order, and the beam divergence (which is here a convolution factor) is the same for the whole reflectivity. From the above we can conclude that the XWG can be used as an excellent beam divergence probe. 
The instrumental broadening can be easily extracted fitting to a theoretical model including smearing with an apparatus function. In Fig. 4 the experimental reflectivity obtained from the carbon structure used as a resolution probe is compared with the result of fitting with resolution parameter $\Delta=0.0014^{\circ}$. The wavelength was set to $\lambda=1.21175 \AA$. The measurements were done at powder diffractometer installed at B2 synchrotron beamline in HASYLAB (Hamburg) and proved applicability of this device to reflectometric measurements [7].

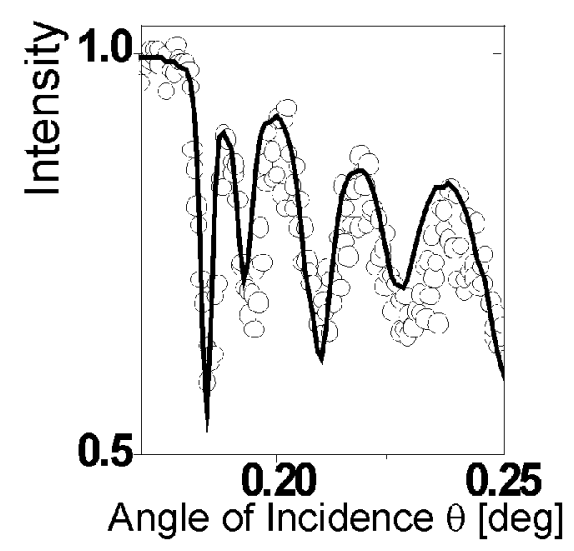

Fig. 4. Reflectivity of thin carbon probe film measured at B2 powder diffractometer station in HASYLAB, Hamburg. Open circles - experimental points, solid line - result of fitting with the resolution parameter $\Delta=0.0014^{\circ}$. The wavelength was set to $\lambda=$ $1.21175 \AA$.

It is also possible to obtain the values of the beam divergence with a reasonable accuracy simply by comparing the experimental intensity ratio in the first minima with pre-tabulated values.

\subsection{Wavelength and angular calibration with $X W G$}

XWG reflectivity with its extremely sharp and narrow minima can be also useful in calibration of the wavelength. In Fig. 5 a calculated intensity distribution map in $\lambda, \theta$ ( $\lambda, \theta$ - wavelength, angle of incidence, respectively) coordinates is shown. The narrow feature observed near the wavelength $\lambda \approx 1.49 \AA$ is generated by a strong variation of the optical constants near the absorption edge of Ni layer in the waveguide structure. The calculated pattern gives evidence that the angular positions of minima almost linearly increase with wavelength. Our experience supported by calculations shows that from the experimental positions of the minima we can easily deduce the wavelength with relative accuracy better than $10^{-4}$, which is usually sufficient in the most of scattering experiments.

In a similar way, also with reasonable accuracy, the angular positions can be calibrated. It has to be stressed that both kinds of calibration can be accomplished 


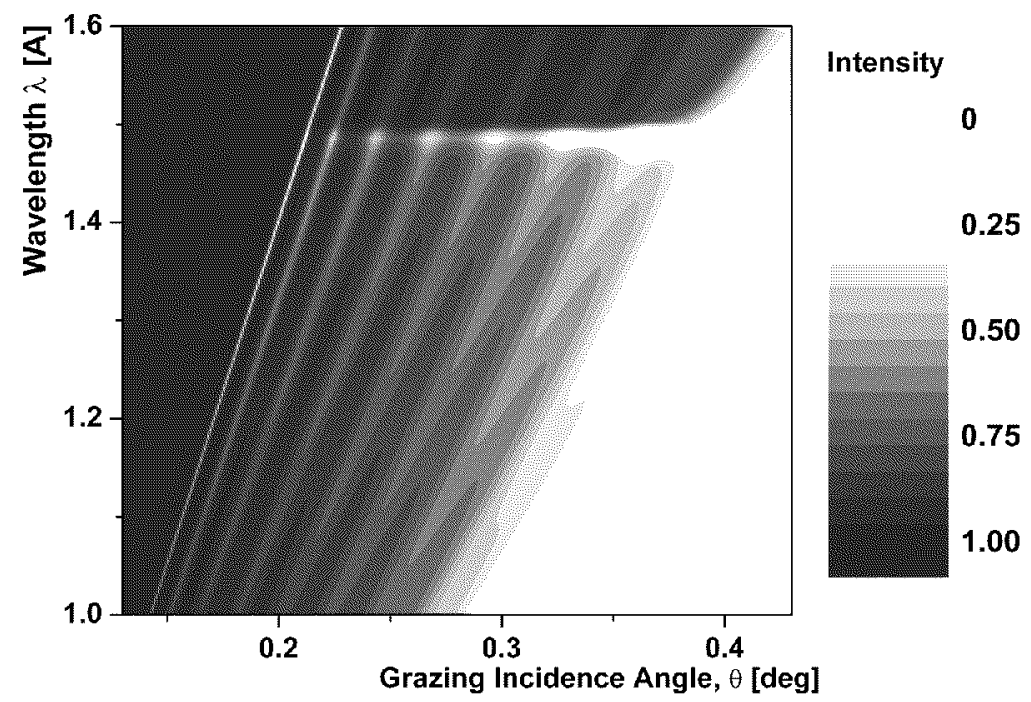

Fig. 5. Calculated intensity distribution map in $\lambda, \theta(\lambda, \theta-$ wavelength, angle of incidence, respectively) coordinates for the quasi-XWG structure composed of $95 \mathrm{~nm}$ graphite film deposited on nickel.

even if we do not know the zero of the angular scale; it can be deduced from the minima positions of the reflectivity.

\section{Acknowledgments}

This work was partially supported by the State Committee for Scientific Research, grant No. 2P 03B 095 16. A part of the synchrotron measurements has been done at HASYLAB, Hamburg and was supported by the IHP-Contract HPRI-CT-1999-00040 of the European Community.

\section{References}

[1] A. Cedola, S. Lagomarsino, S. Di Fonzo, W. Jark, C. Riekel, P. Deschamps, J. Synchrotron Radiat. 5, 17 (1998).

[2] W. Jark, S. Di Fonzo, S. Lagomarsino, A. Cedola, E. Di Fabrizio, A. Bram, C. Riekel, J. Appl. Phys. 80, 4831 (1996).

[3] S. Di Fonzo, W. Jark, S. Lagomarsino, A. Cedola, B. Muller, J.B. Pełka, Thin Solid Films 287, 288 (1996).

[4] J. Wang, M.J. Bedzyk, T.L. Penner, M. Caffrey, Nature 354, 377 (1991).

[5] D.K.G. De Boer, Phys. Rev. B 44, 498 (1991).

[6] L.G. Parratt, Phys. Rev. 95, 359 (1954).

[7] J.B. Pełka, W. Paszkowicz, M. Brust, C.J. Kiely, M. Knapp, in: HAS YLAB Jahresbericht 2000, Vol. I, Eds. W. Dix, T. Kracht, U. Krell, G. Materlik, J.R. Schneider, HASYLAB, Hamurg 2000, p. 461. 